\title{
Modeling and QoS Analysis of the IEEE 802.11p Broadcast Scheme in Vehicular Ad Hoc Networks
}

\author{
Baozhu Li, Gordon J. Sutton, Bo Hu, Ren Ping Liu, and Shanzhi Chen
}

\begin{abstract}
Quality of Service (QoS) and queue management are critical issues for the broadcast scheme of IEEE 802.11p systems in Vehicular Ad hoc Networks (VANETs). However, existing 1dimensional (1-D) Markov chain models of 802.11p systems are unable to capture the complete $\mathrm{QoS}$ performance and queuing behavior due to the lack of an adequate finite buffer model. We present a 2-dimensional (2-D) Markov chain that integrates the broadcast scheme of the 802.11p system and the queuing process into one model. The extra dimension, which models the queue length, allows us to accurately capture the important QoS measures, delay and loss, plus throughput and queue length, for realistic 802.11p systems with finite buffer under finite load. We derive a simplified method to solve the steady state probabilities of the 2-D Markov chain. Our 2-D Markov chain model is the first finite buffer model defined and solved for the broadcast scheme of 802.11p systems. The 2-D model solutions are validated by extensive simulations. Our analyses reveal that the lack of binary exponential backoff and retransmission in the 802.11p system results in poor QoS performance during heavy traffic load, particularly for large VANETs. We demonstrate that our model provides traffic control guidelines to maintain good QoS performance for VANETs.
\end{abstract}

Index Terms: IEEE 802.11p, Broadcast Scheme, VANET, 2-D Markov Chain.

\section{INTRODUCTION}

$\mathrm{T}$ RAFFIC congestion has been increasing worldwide as a result of increased motorization, urbanization, population growth, and changes in population density. Congestion reduces efficiency of transportation infrastructure and increases travel time, air pollution, and fuel consumption. Intelligent Transport Systems (ITS) aims at providing innovative traffic management services that enable road users to be better informed. Vehicular Ad-hoc Networks (VANETs), as an important component of ITS, has attracted significant attention from both industry and research communities [1].

In recent years, a novel type of wireless access called Wireless Access for Vehicular Environment (WAVE) [2] has been proposed, which is dedicated to vehicle-to-vehicle and vehicle-toroadside communications. Also dedicated short-range commu-

Manuscript submitted December 2013. This work has been published in part at the IEEE International Conference on Communications (ICC), June 2013[1].

B. Li and B. Hu are with State Key Laboratory of Networking and Switching Technology, Beijing University of Posts and Telecommunications, Beijing, China (e-mail: \{tiger_1984, hubo\} @bupt.edu.cn)

G.J. Sutton and R.P. Liu are with the Global Big Data Technologies Centre, University of Technology Sydney, Australia (e-mail: \{gordon.sutton, RenPing.Liu\}@uts.edu.au). G.J. Sutton is also with the School of Chemistry, The University of New South Wales, Sydney, NSW, 2052, Australia.

S. Chen is with State Key Laboratory of Wireless Mobile Communications, China Academy of Telecommunications Technology(CATT), Beijing, China. nication (DSRC) [3], [4] has been standardized to support both public safety applications and private applications in VANETs. Moreover, an IEEE task group working on the IEEE 802.11p standard has amended the IEEE 802.11 standard to support VANET [5].

In VANETs with high-speed vehicles and frequent topology changes, broadcast has been proved an effective message delivery mode [6], [7], [8]. Additionally, route messages are exchanged through broadcasts periodically between neighboring vehicles to establish routes, such that congestion and/or emergency messages can be relayed to avoid further delay or damages when an accident happens [9]. In addition, most network services (e.g., address resolution protocol, dynamic host configuration protocol) also use some form of broadcast/multicast communication.

We propose a 2-D Markov chain queueing model with finite buffer under finite load to characterize the IEEE $802.11 \mathrm{p}$ broadcast scheme for VANETs. In our 2-D model, the $1^{\text {st }}$ dimension represents the backoff process and the $2^{\text {nd }}$ dimension embodies the queueing process. Compared with previous 1-D models, we introduce a new dimension to characterize the buffer occupancy. This extra dimension allows us to eliminate the impractical bufferless or infinite buffer assumptions of previous 1-D models. Our 2-D model can be seen as a transformation of the 3-D WLAN Markov model of [10] into the 2-D VANET Markov model proposed here. The proposed 2-D Markov chain is solved by adapting the CTB method of [10] to VANET. Analytical results, validated by simulations, demonstrate that our 2-D model accurately characterizes network performance such as collision probability and throughput. Furthermore, the extra dimension allows us to obtain practical QoS measures, such as queue length, packet delay, and queue blocking probability, which are difficult to achieve with existing 1-D models.

Our model results provide a number of interesting insights into VANET protocol behavior. Our results and analysis show that the broadcast traffic reaches saturation at higher offered load than that of unicast traffic due to the lack of retransmission. When operating under saturation, due to the absence of binary exponential backoff, the broadcast traffic achieves worse performances, i.e. higher collision, lower throughput, higher delay and higher packet loss, than that of unicast traffic. Additionally, the performance degradations are worse for larger networks due to higher contention levels from a large number of nodes and the lack of exponential backoff. Our analysis reveals that such high contention results in significant waste of the radio resources. Our model points to a Network traffic load threshold beyond which the network QoS degrades. Such threshold can be used to provide traffic control guidelines to maintain VANET QoS performance. As put in [11], "evaluating messaging 
reliability and designing mechanisms to reduce interference and congestion requires an in-depth understanding of MAC performance under congestion". Our modeling directly adds to this in-depth understanding.

The remainder of the paper is organized as follows. Related works are discussed in Section II. In Section III we present a brief overview of the broadcast service of IEEE 802.11p in the context of VANET. In Section IV we introduce our proposed 2-D Markov chain queueing model. In Section V we present a simplified method to solve the 2-D Markov model. In Section VI we analyze the performance of the broadcast scheme in VANET with model and simulation results. Concluding remarks are given in Section VII.

\section{RELATED WORK}

Significant work has been done on the design and analysis of VANETs. In [12], $\mathrm{Xu}$ et al. designed several random access protocols for medium access control in single-hop broadcast to improve reception reliability and channel throughput. In [13], Torrent-Moreno et al. addressed the question of how well a priority mechanism was able to work in IEEE 802.11-based vehicular ad hoc networks and showed that the broadcast reception probability can become very low under saturation conditions. In [14], Jiang et al. provided an overview of DSRC based vehicular safety communications and proposed a coherent set of protocols to address channel congestion control and broadcast performance improvement for vehicular safety communication. In [15], ElBatt et al. discussed the suitability of DSRC periodic broadcast message for cooperative collision warning application and explored two design issues, namely performance trends with distance and potential avenues for broadcast enhancement. However, all these analyses and observations are based on simulations.

Theoretical analysis of VANETs is mainly based on the Markov chain model pioneered by Bianchi. Bianchi's 2-D Markov chain model [16] successfully characterised the performance of the IEEE 802.11 MAC protocol under the assumptions of saturated traffic conditions and infinite buffer size. Subsequently, there are a number of extensions of Bianchi's model. Malone et al. [17] added post-backoff states to Bianchi's model to analyse the unsaturated traffic networks under a bufferless assumption. Liu et al. proposed a 3-D Markov chain model with finite buffer under unsaturated traffic load. This 3-D model characterises the QoS measures of IEEE 802.11 networks.

Markov chain analyses have been applied to VANETs. In [18], saturation performance of the broadcast scheme in IEEE 802.11 was studied by constructing an analytical model to characterize the operation of the backoff counter for broadcast service. They divided the backoff counter process into two sub-processes and constructed hierarchical models to calculate a transmission probability in each slot. Then, they adopted the concept of a virtual slot to combine two sub-process models, for the channel probability and freeze length expectation, so that closed-form expressions of typical saturation performance for broadcast could be derived. They pointed out that analytical models for saturation performance evaluation of IEEE 802.11 unicast communication cannot simply be reduced for the analysis of broadcast service and gave constructive suggestions to improve the performance of the broadcast service.

In [19], Ma et al. constructed a 1-D Markov chain model with two levels of safety services to characterize the backoff counter process of each vehicle in IEEE 802.11 broadcast network. Then they applied the proposed model to evaluate the impact of the message arrival interval, channel access priority schemes, the hidden terminal problem, fading transmission channels, and highly mobile vehicles on transmission delay and packet reception rates. In an extended work [6], they constructed a 1-D Markov analytical model to evaluate performance and reliability indices such as channel throughput, transmission delay, and packet reception rates of a typical network solution for DSRCbased safety-related communication under highway wireless communication environment. From the obtained numerical results under various offered traffic and network parameters, new insights and enhancement suggestions were given.

In [20], each node was modeled as a discrete time G/G/1 queue, by which the queueing delay and queue length characteristics were evaluated in an IEEE 802.11 MAC based wireless network. In [7], an analytic model that combined a discrete time M/G/1 queue with a 1-D Markov model was presented to evaluate the performance indices of IEEE 802.11 ad hoc broadcast networks such as packet delay, throughput, packet delivery ratio, and service time distribution in safety related vehicle-to-vehicle (V2V) communication. The discrete time M/G/1 queue modeled occasional occurrences of safety related messages in each vehicle and the 1-D Markov chain modeled the backoff counter process of each station in IEEE 802.11 broadcast network. In [9], a combination of a 1-D Markov model and a discrete time $\mathrm{D} / \mathrm{M} / 1$ queue was presented to analyse the performance of periodic broadcast in VANETs, providing measures such as packet collision probability and average packet delay. In such combined Markov and queueing models, the service time has an unknown complex distribution due to the complicated contention resolution operations. As such, only approximations to the first moment of the service time can be obtained. As a result, these combined models assume an infinite buffer for each VANET node.

In our proposed 2-D Markov chain, the unsaturated load is described by post-backoff states and an idle state. The queueing process is represented by an extra dimension, such that a finite buffer can be characterised. This 2-D Markov chain integrates the $802.11 \mathrm{p}$ contention resolution procedure and queueing process into one model. By solving this 2-D Markov chain, all the VANET QoS measures, system delay and blocking probabilities, plus throughput and collision probability, can be obtained without an explicit service time distribution solution.

\section{BROADCAST SCHEME IN IEEE 802.11P}

The IEEE 802.11p, also known as dedicated short-range communication (DSRC), is a WLAN standard intended for ITS. In order to provide priority-based QoS support, IEEE $802.11 p$ borrowed the ideas of enhanced distributed channel access (EDCA) in IEEE 802.11e as MAC method, which is an enhanced version of the basic distributed coordination function (DCF). EDCA uses carrier sense multiple access with collision avoidance (CSMA/CA) mechanism which is one of the most 


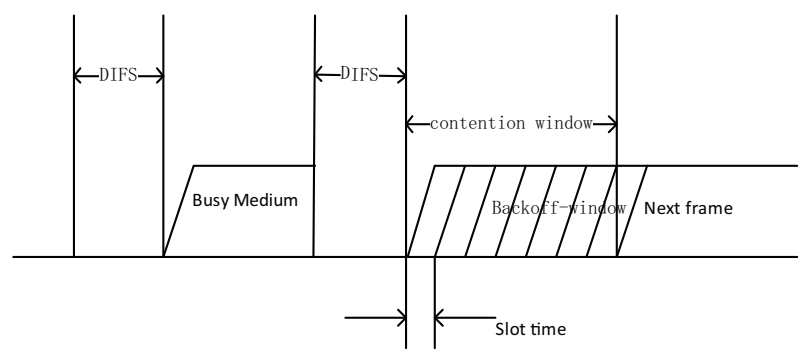

Fig. 1. Basic access mechanism for broadcast.

popular medium access control protocols for Ad Hoc wireless networks designed to reduce collision due to multiple nodes transmitting simultaneously on a shared channel. This means that the node starts by listening to the channel, and if it is idle for an arbitration interframe space (AIFS), the node starts transmitting directly; if the channel is busy or becomes busy during the AIFS, the node must perform a backoff procedure. In the $802.11 \mathrm{p}$ EDCA, there are four available access categories (ACs) with different priorities according to their criticality for the vehicle's safety in a vehicle node: background traffic (BK), best effort traffic (BE), voice traffic (VO) and video traffic (VI). Different AIFS and Contention Window $(\mathrm{CW})$ values are chosen for different ACs.

Broadcast services are widely used in various Ad Hoc network applications especially in VANETs where vehicles with relatively high speed move along roads[4], [21]. In WAVE, urgent messages have to be broadcast over the $\mathrm{CCH}$ during the mandatory periods, so that they can be received by as many devices as possible. Therefore, in the paper we mainly focus on research of QoS and queue management for broadcast scheme in the IEEE 802.11p. For simplicity, we only pay close attention to one of the four available ACs, since all four use the same DCF broadcast scheme. The only difference is the duration of AIFS and the size of $\mathrm{CW}$ as mentioned above.

In broadcast scheme, according to the IEEE $802.11 \mathrm{p}$ protocol, no acknowledgement (ACK) shall be transmitted by any of the recipients of the packet, as replying ACK will cause the ACK explosion problem[22]. When broadcast MAC Protocol Data Units (MPDUs) are transmitted from a station, only the basic access procedure shall be used. There is no MAC-level recovery or retransmission on broadcast frames. As a result, the reliability will be lower in comparison with that in unicast. Additionally, no request-to-send/clear-to-send (RTS/CTS) handshake scheme shall be used regardless of the length of the packet, because CTS has the same explosion problem as ACK. When broadcast data units are transmitted from a node, only the basic access procedure shall be used. Moreover, since a packet collision can not be detected, once the size of the Contention Window, denoted as $W_{0}$, is determined, it will remain constant regardless of whether a packet collides or not. As such, collision can be more serious in broadcast, thus modeling such scheme is important for the success of VANET.

The basic DCF access method for the IEEE 802.11 broadcast scheme is depicted in Fig. 1. Consider a node (i.e., a car) that has an empty queue and is in the idle state. When a new message arrives to broadcast, the node first senses the activities of the channel. If the channel is free for a period of time that exceeds the distributed interframe space (DIFS), the node transmits. Otherwise, if the channel is sensed busy, the node continues to sense the channel until the channel is sensed idle for a DIFS. The node then starts a backoff process and generates a backoff time counter that it selected uniformly in the contention window $\left[0, W_{0}-1\right]$. The backoff time counter is decremented when the channel is sensed idle for a slot time, denoted as $\sigma$, or frozen when the channel is sensed busy, being subsequently reactivated and decremented when the channel is idle for more than a DIFS again. When the backoff time counter reaches zero, the node transmits the packet at the head of the queue. In contrast to the binary exponential backoff scheme in unicast, there is no retransmission in broadcast. After transmission, the node begins another backoff process, again generating a backoff counter uniformly in the contention window $\left[0, W_{0}-1\right]$. If the queue is empty, this process is referred to as post-backoff; otherwise it is referred to as backoff stage- 0 . From post-backoff: if a packet arrives before the backoff counter reaches zero, the packet at the head of the queue is transmitted when backoff counter reaches zero; if instead a packet arrives within $\sigma$ of the backoff counter reaching zero, the packet is immediately transmitted; and otherwise the node returns to the idle state and waits for a new packet to arrive. See IEEE 802.11 standard[23] for details.

\section{THE 2-D MARKOV CHAIN QUEUEING MODEL FOR BROADCAST SCHEME}

\section{A. Assumption}

First of all, we assume that all vehicles in a VANET are equipped with GPS receivers and sensors, from which the states of a vehicle (e.g., location, speed, direction, and acceleration) can be easily obtained. Secondly, we assume that at most only one packet can arrive per time slot, ignoring higher order of arrivals, similar to [17]. And we assume that the collision probability is state-independent which is a common assumption in [20], [10] and [17].

\section{B. Markov Chain Queueing Model}

The 2-D embedded Markov chain ${ }^{1}$ queueing model, which is depicted in Fig. 2, models the broadcast scheme of each node by a 2-tuple $(h, k)$ in conjunction with an idle state, denoted (idle). In the 2-tuple, the index $h$ models the queue occupancy, $0 \leq$ $h \leq L$, where $L$ is the maximum queue length. This allows us to characterize the queueing process more accurately and derive the desired QoS performances such as queue length, blocking probability, queueing delay and so on. The index $k$ models the backoff counter, $0 \leq k<W_{0}$.

Let $q$ be the probability that a packet arrives while in each backoff state, $q_{T}$ be the probability that a packet arrives while in each transmission state and $p$ be the collision probability for the node. In Fig. 2, the top row represents the post-backoff and idle states. The next row of states, indexed $(1,0)$ to $\left(1, W_{0}-\right.$ $1)$, represents the backoff states when there is one packet in the queue. The next row of states, indexed $(2,0)$ to $\left(2, W_{0}-1\right)$, represents the backoff states when there are two packets in the

\footnotetext{
${ }^{1}$ The Markov chain 'embedded' because the states have different durations.
} 


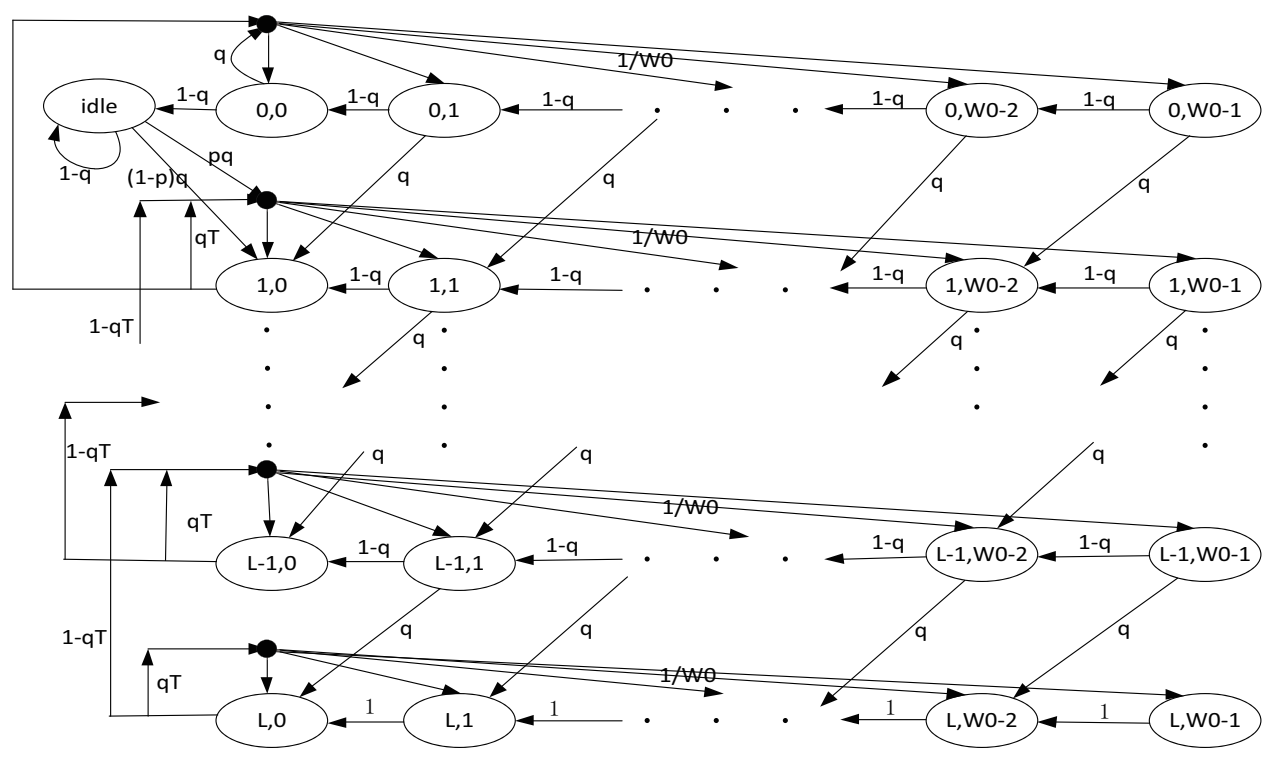

Fig. 2. 2-D Markov Chain Queueing Model for the Broadcast scheme.

queue. And the last row of states, indexed $(L, 0)$ to $\left(L, W_{0}-1\right)$, represents the backoff states when there are $L$ packets in the queue, i.e. the queue is full.

In each backoff state when the index $h$ is less than the queue length $L$ and the index $k$ does not equal 0 , one new packet arrives at the node with probability $q$ before the next counter decrement. This makes the model transition to the state with an increment in index $h=h+1$ and a decrement in index $k=k-1$. Otherwise, the model, with no-packet-arrival probability $1-q$, will enter the state with a fixed value in index $h=h$ and a decrement in index $k=k-1$, which indicates no change in queue occupancy. Describing the above procedure using expressions is as follows: for $0 \leq h<L$ and $0<k<W_{0}$,

$$
\begin{aligned}
& P[(h, k-1) \mid(h, k)]=1-q \\
& P[(h+1, k-1) \mid(h, k)]=q .
\end{aligned}
$$

On the other hand, in the states when the index $h$ equals the queue length $L$ (i.e., the situation that the queue is full) and the index $k$ does not equal 0 , the model transitions to its next state with probability 1 . The expression is as follows:

$$
P[(L, k-1) \mid(L, k)]=1,0<k<W_{0} .
$$

In each backoff state when the index $h$ does not equal 0 and the index $k$ equals 0 (i.e., the state when the packet at the queue head is sent), the packet at the queue head departs from the queue. When transition from this state, the queue occupancy will remain fixed with packet arrival probability $q_{T}$ and will decrease by 1 with the probability $1-q_{T}$ because of no packet arrival, and the index $i$ representing the backoff counter will be uniformly chosen in the range $\left(0 ; W_{0}-1\right)$ inclusive. The procedure can be described as follows: for $0<h \leq L$,

$$
P[(h-1, k) \mid(h, 0)]=\frac{1-q_{T}}{W_{0}}, 0 \leq k<W_{0}
$$

$$
P[(h, k) \mid(h, 0)]=\frac{q_{T}}{W_{0}}, 0 \leq k<W_{0} .
$$

In the state $(0,0)$, if a packet arrives before $\sigma$ seconds elapse, the packet is immediately transmitted, then the node enters postbackoff. We approximate the probability of this occurring as $q$. So, from $(0,0)$, the model transitions to $(i d l e)$, with probability $1-q$, or to one of the states indexed $(0, k), 0 \leq$ $k<W_{0}$, each with probability $q / W_{0}$. It follows therefore that

$$
\begin{gathered}
P[(\text { idle }) \mid(0,0)]=1-q \\
P[(0, k) \mid(0,0)]=\frac{q}{W_{0}}, 0 \leq k<W_{0} .
\end{gathered}
$$

When the model is in the idle state, it remains there with probability $1-q$, because of no packet arrival. When a packet arrives, with probability $q$, the STA performs a carrier sense, and senses a clear channel with probability $1-p$. If the channel is sensed 'clear', the STA transitions to the state $(1,0)$ and immediately begins a transmission attempt. Otherwise it enters a backoff and selects a random initial backoff count uniformly from 0 to $W_{0}-1$. The expressions of the procedure are:

$$
\begin{gathered}
P[(\text { idle }) \mid(\text { idle })]=1-q \\
P[(1,0) \mid(\text { idle })]=(1-p) q+\frac{p q}{W_{0}} \\
P[(1, k) \mid(\text { idle })]=\frac{p q}{W_{0}}, 0<k<W_{0} .
\end{gathered}
$$

This proposed 2-D Markov chain integrates the IEEE 802.11p backoff and the system queueing process, for a vehicular node with a finite queue, into the one model. As such, the model enables us to investigate the complete range of QoS measures, packet delay, average queue length, and queue overflow, in addition to the collision probability and throughput.

\section{SOLUTION TO THE 2-D MARKOV CHAIN QUEUEING MODEL}

In this section we first present the solution to the Markov chain, and then derive the system performance and QoS measures. 


\section{A. Modified CTB Solution}

To solve the new VANET broadcast Markov chain, depicted in Fig. 2, we apply the Collapsed Transition onto Basis (CTB) method presented in [10]. In [10], the CTB method was applied to the 802.11 DCF protocol, which includes the exponential backoff scheme, whereas here we apply the CTB method to the $802.11 \mathrm{p}$ protocol, where each packet is transmitted as a broadcast and only once, regardless of whether the packet is received correctly or not. Using the 802.11 terminology, both modes have post-backoff, backoff stage- 0 , the idle state and carrier sensing. The contrast is that the $802.11 \mathrm{p}$ protocol only has backoff stage-0, since it has no acknowledgement and no retransmissions, whereas the 802.11 DCF protocol additionally has backoff stage-1 to backoff stage- $s$, where $s$ is the maximum number of retransmission attempts. The simpler $802.11 \mathrm{p}$ broadcast system leads to a similar, yet simpler solution.

The steady-state solution to the Markov chain model of Fig. 2 can be obtained via the power method, which involves constructing the $\left(L W_{0}+1\right) \times\left(L W_{0}+1\right)$ transition matrix for the full Markov chain, then successively squaring, starting from the transition matrix, until convergence. With a large matrix, this can be computationally expensive. Instead, we used the CTB method of [10], which collapses parts of the Markov chain, while retaining the correct relative progression probabilities of the remaining states to produce an easily solved Markov chain comprising just $L+1$ states.

We use the same terminology and notation as used in [10]. As such, we define the states $(h, 0), 0 \leq h \leq L$, to be the transmission states, and the states $(h, k), 0 \leq h \leq L, 0<$ $k \leq W_{0}$ to be the backoff stage- 0 states, reiterating that there is only the one backoff stage, stage- 0 . Let $\mathcal{V}$ be the ordered set of transmission states $(0,0), . .,(L, 0)$, and $\mathbf{v}$ be the associated steady-state probabilities of $\mathcal{V}$.

The approach in [10], for the 802.11 DCF protocol, proceeds by following the progression of the system from the initial transmission attempt for a particular packet through to the packet's completion, which may involve multiple backoff stages, then through post-backoff/backoff stage-0 to the next packet's initial transmission attempt. The progression of the system through the idle state is also followed as a separate loop. The process is the same for the $802.11 \mathrm{p}$ protocol except there is only backoff stage- 0 .

In particular, in [10] for the 802.11 DCF protocol, the matrix $\mathbf{B}$ contains the progression probabilities from $\mathcal{V}$ onto the set of transmission states. For the $802.11 \mathrm{p}$ protocol, the set of transmission states is simply $\mathcal{V}$, so $\mathbf{B}$ equals the identity matrix and can be omitted. Next, the matrix $\mathbf{C}$ contains the progression probabilities from the set of transmission states onto the 'fan-out' backoff count selectors for backoff stage0 . The 'fan-out' backoff count selectors, as described in [10], are not states, but are transition branching points. The matrix D then contains the progression probabilities from the stage0 'fan-out' backoff count selectors, through backoff stage-0, back onto $\mathcal{V}$. This step effectively collapses all the states in backoff stage- 0 onto the transmission states of backoff stage0 , discarding all information about the particular paths through backoff stage-0, but maintaining the cumulative probabilities of reaching each element of $\mathcal{V}$. Another matrix, denoted $\mathbf{E}$, contains the progression probabilities from $\mathcal{V}$, through the idle state, then directly back to $\mathcal{V}$, or more specifically from $(0,0)$ back to $(1,0)$, without entering backoff; this path results from the channel being sensed clear just after a packet arrives when the node is in the idle state. Combining these steps, we obtain the collapsed $(L+1)$-state Markov chain comprising $\mathcal{V}$, with transition probabilities equal to the progression probabilities between $\mathcal{V}$ in the full Markov chain of Fig. 2. Hence, the relative steady-state probabilities of $\mathcal{V}$ for the collapsed and full Markov chains are proportional. The $(L+1) \times(L+1)$ transition matrix for the collapsed Markov chain is denoted $\mathbf{A}$, such that $\mathbf{v}=\mathbf{A v}$, whence $\mathbf{A}=\mathbf{D C}+\mathbf{E}$.

$\mathbf{C}$ is almost the same as $\mathbf{C}$ obtained from [24], which contains errata to [25], with $i=s=0$. The difference is that $\mathbf{C}[1,1]$ needs modifying to account for broadcasting with no retransmissions, where $\mathbf{C}[i, j]$ is the $i^{\text {th }}$ row and $j^{\text {th }}$ column of C. C is then:

$$
\begin{aligned}
\mathbf{C}[\mathbf{1}, \mathbf{1}] & =q ; \\
\mathbf{C}[\mathbf{2}, \mathbf{1}] & =p(1-q) ; \\
\mathbf{C}[\mathbf{h}, \mathbf{1}+\mathbf{h}] & =\left(1-q_{T}\right), \text { for } h=1, . ., L \\
\mathbf{C}[\mathbf{1}+\mathbf{h}, \mathbf{1}+\mathbf{h}] & =q_{T}, \text { for } h=1, . ., L \\
\mathbf{C}[\mathbf{i}, \mathbf{j}] & =0, \text { otherwise. }
\end{aligned}
$$

$\mathbf{D}$ and $\mathbf{E}$ can be obtained directly from [24] and [25] respectively, which are included here for completeness:

$$
\begin{aligned}
& \mathbf{D}[\mathbf{1}+\mathbf{h}, \mathbf{1}+\mathbf{h}-\mathbf{f}]= \frac{q^{f}}{W_{0}} \sum_{j=0}^{W_{0}-1-f}\left(\begin{array}{c}
j+f \\
f
\end{array}\right)(1-q)^{j} \\
& \text { for }\left\{\begin{array}{l}
h=0, . ., L-1, \\
f=0, . ., \min \left(h, W_{0}-1\right)
\end{array}\right. \\
& \mathbf{D}[\mathbf{i}, \mathbf{j}]= 0, \text { otherwise, for } i \neq L+1 ; \text { and } \\
& \mathbf{D}[\mathbf{L}+\mathbf{1}, \mathbf{j}]=1-\sum_{h=1}^{L} \mathbf{D}[\mathbf{h}, \mathbf{j}], \mathbf{j}=\mathbf{1}, . ., \mathbf{L}+\mathbf{1}
\end{aligned}
$$

and

$$
\mathbf{E}[\mathbf{i}, \mathbf{j}]=\left\{\begin{array}{l}
(1-p)(1-q), i=2, j=1 \\
0, \text { otherwise }, i, j=1, . ., L+1
\end{array}\right.
$$

To obtain $\mathbf{v}$, we first solve the vector of steady-state probabilities for the collapsed system, denoted $\tilde{\mathbf{v}}$, which equals the eigenvector of $\mathbf{A}$ with eigenvalue 1 that has $\|\tilde{\mathbf{v}}\|_{1}=1$, where $\|\cdot\|_{1}$ is the 1 -norm. Note that $\tilde{\mathbf{v}}$ can be readily obtained for the relatively small $(L+1) \times(L+1)$ matrix $\mathbf{A}$. We then normalise $\tilde{\mathbf{v}}$ by $c$ to give $\mathbf{v}=\tilde{\mathbf{v}} / c$, where $c$ is the normalising constant that ensures the sum of the steady-state probabilities of the full Markov chain equals 1 . That $\mathbf{v}$ is unique is proved in [25].

To calculate the normalisation constant $c$, we sum the probabilities of being in each state of the full Markov chain, conditional on being in $\mathcal{V}$ with probabilities $\tilde{\mathbf{v}}$. Conditional on $\tilde{\mathbf{v}}$, the probability of reaching one of the backoff stage- 0 backoff count selectors is $\|\mathbf{C} \tilde{\mathbf{v}}\|_{1}$, whence there are many different paths through backoff stage- 0 ; on average these paths comprise $\left(W_{0}+1\right) / 2$ transitions. The idle state is only reached from state $(0,0)$, with transition probability $1-q$, and once the idle state is 
entered, the average number of transitions spent before leaving it is $1 / q$. So, we have:

$$
c=\|\mathbf{C} \tilde{\mathbf{v}}\|_{1}\left(W_{0}+1\right) / 2+\frac{1-q}{q} \tilde{\mathbf{v}}[1,1] .
$$

This uniquely determines the steady-state probabilities of the embedded Markov chain of Fig. 2, given the parameters $p, q$ and $q_{T}$. We can then obtain the state probability that the particular vehicle is transmitting, denoted $\tau$, as:

$$
\tau=\|\mathbf{v}\|_{1}-(1-q) \mathbf{v}[1,1],
$$

noting that 'state probability' refers to the embedded Markov chain probabilities, in which each state is given equal weight. We will later need the time probabilities, in which each state is weighted by its average duration. The time probabilities will be used to determine the Markov chain parameters, $p, q$ and $q_{T}$, and to evaluate the throughput and QoS measures.

\section{B. State-to-time Conversion}

To convert the Markov chain state probabilities to time probabilities, each state is weighted by its average duration and normalised by the average state duration. In this section we calculate the average duration for each state and the average duration over all the states.

We assume a symmetric network, in which all vehicles have the same offered load and follow the IEEE 802.11p protocol. We assume a perfect channel, with no noise and no fading, and that all vehicles in the network can hear transmissions made by other vehicles in the network. Since all vehicles follow the IEEE $802.11 \mathrm{p}$ protocol, the network counts are synchronised such that all the vehicles make simultaneous state transitions (e.g. backoff countdowns, or idle-state transitions). We assume that all vehicles broadcast the same sized packets and that the state duration of a broadcast is $T_{b}$. That is, when any vehicle is broadcasting, the current state duration for all vehicles is $T_{b}$. When no vehicle is broadcasting, the state duration is instead a slot time, which we denote $\sigma$.

Let $n$ denote the number of vehicles in the network. Recalling that $\tau$ is the state probability that a particular vehicle is broadcasting, the state probability that none of the $n$ vehicles are broadcasting, which we denote $P_{e}$, is $P_{e}=(1-\tau)^{n}$. Hence, the state probability that at least one vehicle is broadcasting, which we denote, $P_{b}$, is $P_{b}=1-P_{e}$. The average state duration, which we denote $E_{s}$, is then

$$
E_{s}=P_{e} \sigma+P_{b} T_{b}
$$

As explained in [10], the average duration for states in which the vehicle is not transmitting, denoted $E_{s \mid n-1}$, is equivalent to $E_{s}$ for an (n-1)-vehicle system, due to vehicle independence. Thus:

$$
E_{s \mid n-1}=P_{e \mid n-1} \sigma+P_{b \mid n-1} T_{b},
$$

where $P_{e \mid n-1}=(1-\tau)^{n-1}$ and $P_{b \mid n-1}=1-P_{e \mid n-1}$.

\section{Markov chain parameters}

The Markov chain models a single node in the system. The nodes of the system are dependent though because they share the same channel, which causes collisions. When a particular vehicle broadcasts, a collision will occur if at least one other vehicle is also broadcasting. As such, for a symmetric $n$-vehicle system, the collision probability, $p$, is:

$$
p=1-(1-\tau)^{n-1} .
$$

$p$ also equals the carrier sense probability that the channel is sensed 'busy'. We quantify the offered load for the network as was done in [10], and define the normalised offered load, denoted $\lambda$, as the expected number of packets delivered to all $n$ nodes per time for a broadcast. We also define $r_{q}$ as the average packet arrival rate per second at each node. Thus:

$$
\begin{gathered}
\lambda=n r_{q} T_{b}, \\
q_{T}=r_{q} T_{b}
\end{gathered}
$$

and

$$
q=r_{q} E_{s \mid n-1} .
$$

We wish to find the solution to a symmetric $n$-vehicle system, with maximum queue length $L$ that is operating with a normalised offered load $\lambda$. For a given set of $p, q$ and $q_{T}$, solving the Markov chain gives $\tau$ and in turn, $E_{s \mid n-1}$. Conversely, (15)(18) provide equations for $p, q$ and $q_{T}$, based on $\tau, E_{s \mid n-1}$ and network interactions. We use the following iterative procedure, similar to that in [10], to find the set of $p, q, q_{T}$ and $\tau$ that simultaneously satisfies the Markov chain relationships and the network interaction equations:

- define $n, L$ and $\lambda$ of interest;

- select a candidate $\tau$;

- calculate $p, q$ and $q_{T}$ from (13)-(18);

- solve the Markov chain queueing model for $p, q$ and $q_{T}$, as in Section $\mathrm{V}$, and obtain $\tau$ from (12);

- compare the Markov chain $\tau$ to the candidate $\tau$

- if discrepancy is small enough, stop;

- else, decide next candidate $\tau$ based on a search method and loop.

\section{Network Properties}

Once the single-vehicle Markov chain and network interaction models are simultaneously solved, we can calculate various network properties and QoS measures. In particular, again using the notation from [10], we can calculate:

- $S$, the throughput, or proportion of time the network spends successfully transmitting payload data;

- $\bar{Q}$, the average interface queue occupancy (at arrival times);

- $P_{B}$, the queue blocking probability (or proportion of offered load lost due to the input buffer being full); and

- $D_{t}$, the total system delay (or the average time from when a packet first joins the input interface queue till it is successfully transmitted).

As in [10], $S$ can be calculated as:

$$
S=P_{s} E_{p} / E_{s}
$$

where $E_{p}$ is the expected time spent transmitting payload data during each successful transmission and $P_{s}$ is the network stateprobability of a successful transmission, given by:

$$
P_{s}=n \tau(1-p) \text {. }
$$


To determine $\bar{Q}$, we follow the derivation in [10], Section III.E, adapting it to the broadcasting scenario, which results in slightly different equations. The steps involved are:

1. calculate the state probability of being in states with queue occupancy $h$, denoted $P_{s}[Q=h]$, for $0 \leq h \leq L$;

2. split each $P_{s}[Q=h]$ into transmitting and non-transmitting components by calculating the state probability of broadcasting with queue occupancy $h$, denoted $P_{s}\left[Q=h \cap \mathrm{T}_{\mathrm{x}}\right]$, for $0 \leq h \leq$ $L$;

3. weight the two components for each queue length by the average duration of the states in the component (either $T_{b}$ or $\left.E_{s \mid n-1}\right)$ and normalise by the node-average state duration, to give the time probability of having queue occupancy $h$, denoted $P[Q=h]$, for $0 \leq h \leq L$;

4. obtain $\bar{Q}$ as the weighted average of $P[Q=h]$.

To find $P_{s}[Q=h]$ for Step 1), we first define $\mathbf{H}_{\mathbf{0}}$, as in [10], which is the $(L+1) \times(L+1)$ matrix that maps the state probabilities of reaching the stage- 0 backoff count selectors onto the summed state probabilities of being in states with queue length $h, 0 \leq h \leq L$, during backoff stage- 0 . $\mathbf{H}_{\mathbf{0}}$ can be obtained from [24] by setting $i=0$, which becomes:

$$
\begin{aligned}
& \mathbf{H}_{\mathbf{0}}[1+h+f, h+1] \\
& =\frac{1}{W_{0}} \sum_{k=f}^{W_{0}-1} \sum_{r=0}^{k-f}\left(\begin{array}{c}
r+f \\
f
\end{array}\right)(1-q)^{r} q^{f}, \\
& \text { for }\left\{\begin{array}{l}
h=0, . ., L-1, \\
f=0, . ., \min \left(L-1-h, W_{0}-1\right) \text {; }
\end{array}\right. \\
& \mathbf{H}_{\mathbf{0}}[j, k]=0 \text {, otherwise, for } j \neq L+1 \text {; } \\
& \mathbf{H}_{\mathbf{0}}[L+1, k]=\frac{W_{0}+1}{2}-\sum_{j=1}^{L} \mathbf{H}_{\mathbf{0}}[j, k], \text { for } k=1, . ., L+1 \text {. }
\end{aligned}
$$

Then, including non-backoff contributions to $P_{s}[Q=h]$, which comprise being in (idle) and transmitting in $(1,0)$ directly after a packet arrives in $(i d l e)$ and the channel is sensed 'free', we have:

$$
\begin{aligned}
P_{s}[Q=h]= & \left(\mathbf{H}_{\mathbf{0}} \mathbf{C v}\right)[h+1,1]+\delta_{0 h} \frac{1-q}{q} \mathbf{v}[\mathbf{1}, \mathbf{1}] \\
& +\delta_{1 h}(1-p)(1-q) \mathbf{v}[1,1]
\end{aligned}
$$

where $\delta_{x y}$ is the kronecker delta function and equals 1 , if $x=y$, and otherwise 0. $P_{s}\left[Q=h \cap \mathrm{T}_{\mathrm{x}}\right]$, for Step 2), comes directly from $\mathbf{v}$, noting a correction is needed for $(0,0)$, giving:

$$
P_{s}\left[Q=h \cap \mathrm{T}_{\mathrm{x}}\right]=\mathbf{v}[h+1,1]-\delta_{0 h}(1-q) \mathbf{v}[1,1] .
$$

Weighting broadcasting states by $T_{b}$ and non-broadcasting states by $E_{s \mid n-1}$, and normalising by $E_{s}$, gives $P[Q=h]$ as:

$$
\begin{aligned}
P[Q=h]= & \left(\left(P_{s}[Q=h]-P_{s}\left[Q=h \cap \mathrm{T}_{\mathrm{x}}\right]\right) E_{s \mid n-1}\right. \\
& \left.+P_{s}\left[Q=h \cap \mathrm{T}_{\mathrm{x}}\right] T_{b}\right) / E_{s} .
\end{aligned}
$$

Then, finally, $\bar{Q}$ is:

$$
\bar{Q}=\sum_{h=0}^{L} h P[Q=h] .
$$

The states $(L, 0), \ldots,\left(L, W_{0}-1\right)$ represent when the queue is full. In these states, if a new packet arrives it is blocked, except for $(L, 0)$. For $(L, 0)$, the packet being transmitted is removed from the queue, in time to allow a new arrival. Thus,

$$
P_{B}=P[Q=L]-\mathbf{v}[L+1,1] T_{b} / E_{s} .
$$

$D_{t}$ can be calculated as the sum, over all states in which an arriving packet can join the input interface queue, of the time-probability of the packet arriving in that particular state, given the packet is successfully transmitted at some point, times the average time from the packet's arrival till its completed transmission, given it arrives in that state and is successfully transmitted. That is:

$$
D_{t}=\frac{\sum_{k=0}^{W_{0}-1} \sum_{h=0}^{L-1+\delta_{k 0}} a((h, k)) d((h, k))+a((\text { idle })) d((\text { idle }))}{\sum_{k=0}^{W_{0}-1} \sum_{h=0}^{L-1+\delta_{k 0}} a((h, k))+a((\text { idle }))} .
$$

where $a($ state $)$ is the probability that a packet arrives in the state state and is successfully transmitted at some point; and $d$ (state) is the average time to successfully transmit a packet that arrived in the state state.

To evaluate the components of $D_{t}$, we define $\Lambda$ as the average time from the initiation of a new backoff stage- 0 countdown, given that there is a packet in the queue, till the packet at the head of the queue has been broadcast. Thus,

$$
\Lambda=T_{b}+\frac{W_{0}-1}{2} E_{s \mid n-1} .
$$

Under the perfect channel assumption, the probability that a packet in the queue is successfully transmitted at some point is $1-p$, regardless of the path to the transmission attempt. Hence, $\Lambda$ is the time till both a successful and an unsuccessful broadcast occurs.

For $(i d l e)$, there are two cases. The packet is either directly broadcast, with probability $1-p$, after the channel is sensed 'clear', or otherwise broadcast after a backoff countdown. If directly broadcast, the processing time is $T_{b}$; if broadcast after a backoff countdown, the average processing time is $\Lambda$. The time probability of being in $(i d l e)$ is $\frac{1-q}{q} \mathbf{v}[1,1] \frac{E_{s \mid n-1}}{E_{s}}$.

For post-backoff states, if a packet arrives during the backoff countdown, it is broadcast at backoff count 0 . Let the sum contributions from post-backoff states to the numerator of $D_{t}$ in (26), be $d_{p b}$. To obtain $d_{p b}$, we convert the state probabilities of a packet arriving in each post-backoff state and being successfully broadcast to time probabilities, then weight each state by the average time to complete the broadcast from this state. After some working we obtain

$$
\begin{aligned}
d_{p b}= & \frac{(\mathbf{C v})[1,1]}{W_{0}} \frac{E_{s \mid n-1}}{E_{s}}(1-p) \sum_{k=0}^{W_{0}-1}\left(1-(1-q)^{k+1}\right) \\
& {\left[T_{b}+\left(W_{0}-k-\frac{1}{2}\right) E_{s \mid n-1}\right] . }
\end{aligned}
$$

For backoff states (i.e. with non-empty queue) that also have a non-full queue, we approximate the time to process the packet that is head-of-the-queue upon the new packet's arrival as $\Lambda / 2$. 
Table 1. Simulation Settings

\begin{tabular}{ll}
\hline Parameter & Value \\
\hline Timeslot size, $\sigma$ & $20 \mathrm{us}$ \\
DIFS & $50 \mathrm{us}$ \\
Propagation delay, $\delta$ & $1 \mathrm{us}$ \\
Basic rate, $b r$ & $1 \mathrm{Mbit} / \mathrm{s}$ \\
Transmission rate, $t r$ & $1 \mathrm{Mbit} / \mathrm{s}$ \\
$C W$ size, $W_{0}$ & 32 \\
Preamble+PLCP_Hdr, $L_{p}$ & $144+48$ bits \\
MAC_Hdr+FCS, $L_{H}$ & $30+4$ bytes \\
Data payload, $L_{P}$ & 64 bytes
\end{tabular}

After this, there are $h$ packets to fully process, including the new packet. Each of these $h$ packets in the queue then takes $\Lambda$ on average to process. Hence, the average processing time for packets arriving in backoff states with $0<h<L$, is approximated as $(h+1 / 2) \Lambda$.

For backoff states with $h=L$, packets can only join the queue in $(L, 0)$, where a spot in the queue is freed by the broadcasting process. After the current broadcast, there are $L$ full packets to process. The time probability of a packet joining the queue in $(L, 0)$ is $\mathbf{v}[L+1,1] T_{b} / E_{s}$, and the average processing time is $T_{b} / 2+L \Lambda$.

Putting this all together to evaluate (26), we have:

$$
\begin{aligned}
D_{t}= & {\left[\frac{1-q}{q} \mathbf{v}[1,1] \frac{E_{s \mid n-1}}{E_{s}}\left((1-p) T_{b}+p \Lambda\right)\right.} \\
& +\frac{d_{p b}}{1-p}+\sum_{h=1}^{L-1} P[Q=h]\left(h+\frac{1}{2}\right) \Lambda \\
& \left.+\mathbf{v}[L+1,1] \frac{T_{b}}{E_{s}}\left(\frac{T_{b}}{2}+L \Lambda\right)\right] /\left(1-P_{B}\right),
\end{aligned}
$$

noting that since the probability that the packet is broadcast successfully is $1-p$ for all contributing states, it cancels out.

\section{SIMULATIONS AND PERFORMANCE ANALYSIS}

Extensive simulations were conducted in ns-2 to validate the accuracy of our 2-D Markov chain queueing model. The ns2802.11 DCF module were modified to simulate the $802.11 \mathrm{p}$ broadcast scheme. All nodes are within communication range of each other, and each node independently generates Poisson traffic with specified rate. Each simulation experiment for a particular network configuration was run for 80 seconds after a 10 second initialisation. The values of interest were calculated for each subsequent 10-second block. The average of the eight 10 -second blocks was taken as one simulation value. We have verified that, with such simulation time, the $95 \%$ confidence intervals for various measures were approximately the same size as the symbols, or smaller, and have hence been omitted in the following figures. The network parameters used in the simulations are given in Table 1.

\section{A. Model validation and performance comparisons}

Fig. 3 shows the collision probability varying with normalized offered load $\lambda$ for different network sizes. The solid, dashed

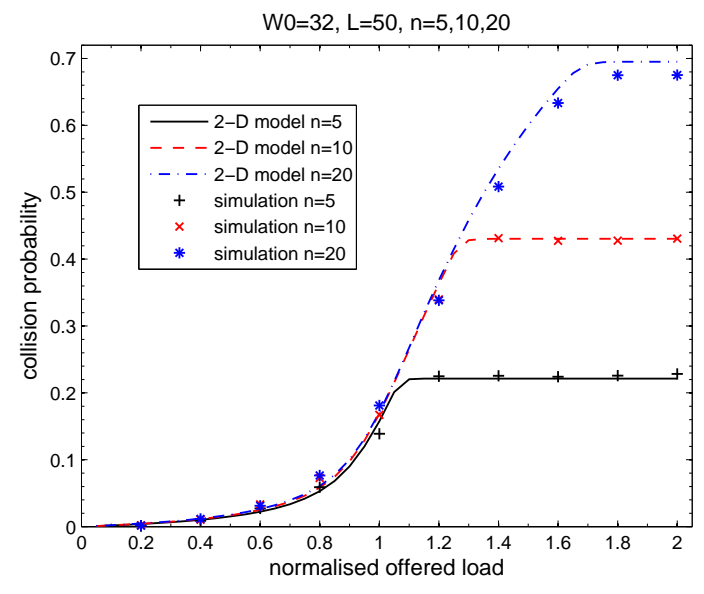

Fig. 3. Collision probability vs offered load over different network sizes.

and dot-dashed curves are for network sizes $n=5,10$ and 20 respectively. It can be seen that there is a very close match between our 2-D model predictions (lines) and the simulation results (markers). At light traffic load, $\lambda<0.5$, the collision probabilities are close to zero. At higher traffic load, $\lambda>$ 0.8 , the collision probabilities rise sharply with $\lambda$, level off at different $\lambda$, and reach different saturation values for different network sizes. The saturated collision probabilities are higher for larger networks due to higher contention levels created by more competing nodes.

Compared with the collision probabilities of unicast traffic, e.g. Fig. 3 of [10], the collision probabilities of broadcast traffic (shown in Fig. 3 here) level off at higher offered loads, and reach higher saturation values. The reason for higher level-off load is that there is no retransmission in the broadcast scheme. The lack of retransmission results in lower effective load for the broadcast scheme, and thus it takes higher offered load to reach saturation. The reason for higher saturation collision probabilities is that there is no binary exponential backoff in the broadcast scheme, i.e. the contention window stays at $W_{0}$ regardless of the network contention level. That is, while the binary exponential backoff in unicast schemes effectively reduces the collision probability, there is no collision avoidance in broadcast scheme. As a result, the saturated collision probabilities for broadcast traffic reach higher saturation values than that of unicast traffic.

Fig. 4 shows the normalised throughput varying with normalized offered load $\lambda$ for different network sizes. We can see again that there is a close match between our 2-D model predictions (lines) and the simulation results (markers). The normalised throughputs rise linearly as the normalized offered loads increase, reaching their peaks at the network capacity $\lambda=1.0$. They then drop down as the collisions rise, leveling off at different levels. The saturated throughput is lower for larger networks. The reason is that the saturated collisions are higher for larger networks as shown in Fig. 3. Such high collisions result in wasted radio resources. Consequently, under saturated conditions, as the network size increases, the collision probability increases, leading to the decrease of the network throughput.

Compared with the throughput of unicast traffic, e.g. Fig. 


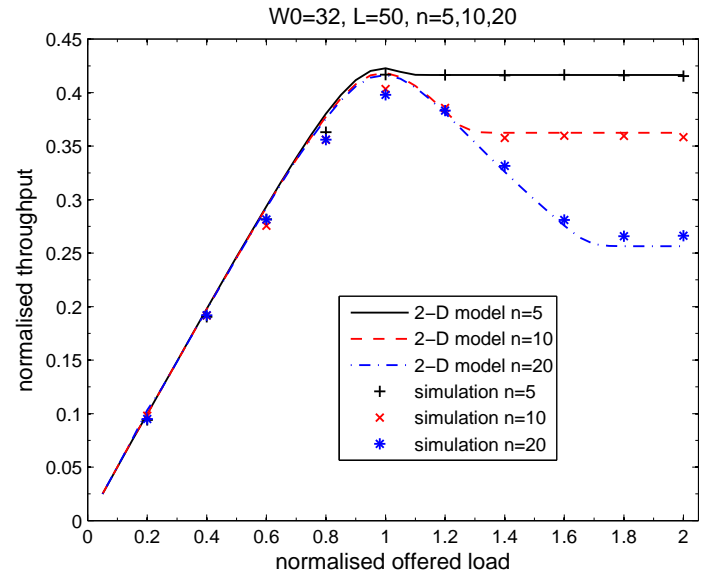

Fig. 4. Normalised throughput vs offered load over different network sizes.

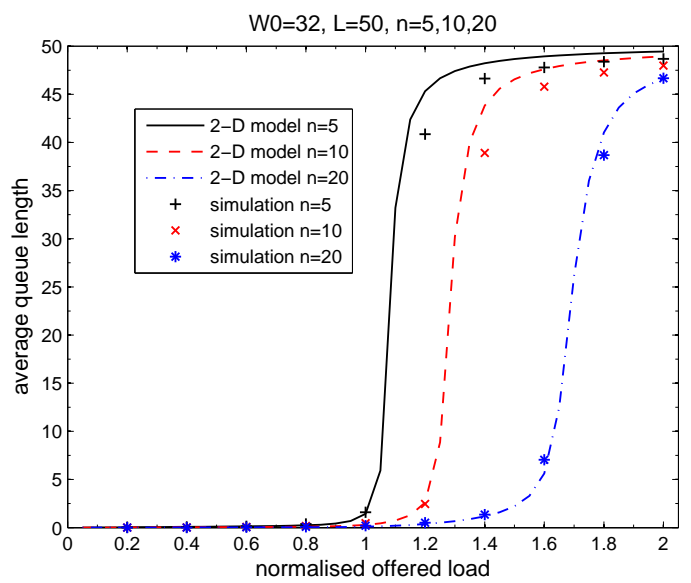

Fig. 5. Average queue length vs offered load over different network sizes.

4 of [10], the throughput of broadcast traffic (shown in Fig. 4 here) level off at higher offered loads, and reach lower saturated throughput. These can be explained by the lack of retransmission and binary exponential backoff, in the similar way as that for Fig. 3.

Another important observation is the existence of a throughput peak similar to that observed in [17], [10] for unicast traffic. However, the throughput peak for broadcast traffic here is more evident, particularly for larger networks.

\section{B. QoS analysis}

In addition to the collision and throughput results, our 2D Markov chain queueing model is able to characterize the QoS measures, including queue length, delay, and blocking probabilities.

Fig. 5 shows the average queue length varying with normalized offered load $\lambda$ for different network sizes. The queue length exhibits an almost bimodal behavior. It is close to zero before saturation, and is almost full when the network is saturated. The switching point from empty to full queue corresponds to the level of offered load where the network reaches saturation, i.e. the level-off points in Figs. 3 and 4.

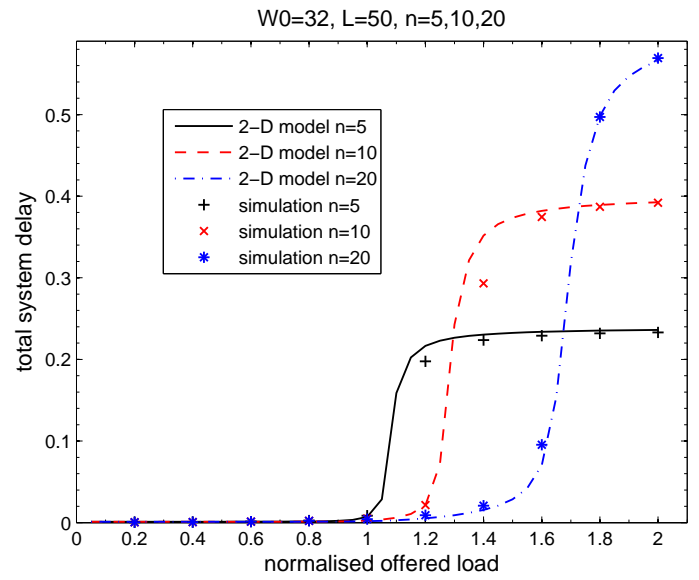

Fig. 6. Average packet delay vs offered load over different network sizes.

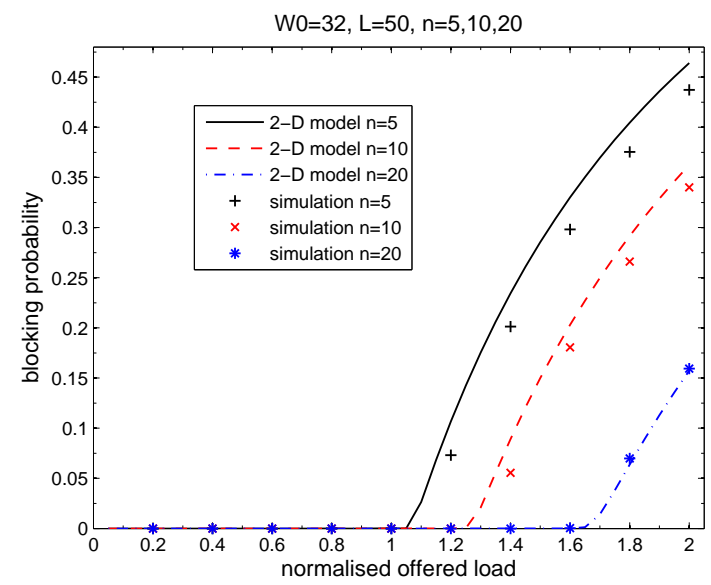

Fig. 7. Blocking probability vs offered load over different network sizes.

Fig. 6 shows the average packet delay varying with normalized offered load $\lambda$ for different network sizes. Not surprisingly, the packet delay also exhibits an almost bimodal behavior, similar to the queue length in Fig. 5. However, it is interesting to note that packet delay reaches different saturation levels for different network sizes: larger networks incur higher packet delay. The reason is that there are more nodes transmitting in a larger network. In such case, a node in a larger network will encounter more freezes in its backoff countdown than the node in a small network. Such frequent freezes prolong the packet backoff count and increase its media access delay. As a result, the packet delay becomes longer for a larger network.

Fig. 7 shows the blocking probability varying with normalized offered load $\lambda$ for different network sizes. The blocking probability can also be related to the queue length of Fig. 5. When the queue length is low, the blocking probability is close to zero. When the queue is high, such that the network is saturated, the blocking probability rises with $\lambda$.

Compared with blocking probabilities of unicast traffic, e.g. Fig. 8 of [10], the blocking probabilities of broadcast traffic (shown in Fig. 7 here) have similar rising trends, although they rise from higher offered loads for broadcast traffic than that 
for unicast traffic. This can be again explained by the lack of retransmission for broadcast in the similar way as that in Fig. 3.

An important observation can be drawn from the performance results: the throughput peaks around the network capacity, i.e. normalised load of $\lambda \approx 1.0$ in Fig. 4 . For loads below this peak, the average queue length, the packet delay, and the queue blocking probability are close to zero, shown in Figs. 5, 6, and 7, for all networks. This points to a traffic load threshold beyond which the network QoS degrades. This threshold can be used to provide traffic control guidelines that maintain VANET QoS performance.

\section{CONCLUSION}

In this paper, we proposed a 2-D Markov chain queueing model for analysing the QoS performance of the IEEE 802.11p broadcast scheme in VANETs. Our 2-D model characterises the unsaturated traffic condition by including an idle state and postbackoff states, and integrates the queueing process with the DCF backoff procedure.

The 2-D Markov chain was solved efficiently by adapting our CTB method to VANETs. Important QoS measures were obtained and validated by extensive simulations. Our analytical results revealed a QoS performance degradation due to the lack of binary exponential backoff and retransmission in VANETs. Such performance deterioration can be avoided by proper traffic control, and our 2-D Markov chain model pointed to a traffic load threshold beyond which the network QoS degrades. This threshold can be used as traffic control guidelines to maintain good QoS performance for VANETs.

\section{ACKNOWLEDGMENTS}

This work is supported by National High-Technology Program (863) of China (No. 2014AA01A701), the Major National Science and Technology Special Project (No. 2013ZX03001025001) and China Next Generation Internet (CNGI) Project (No. CNGI-12-03-003) and partially funded by the National Science Foundation Project (Grant No. 61300183) and the National Science Fund for Distinguished Young Scholars (Grant No. 61425012) in China.

\section{REFERENCES}

[1] B. Li, B. Hu, R. P. Liu, and S. Chen, "Modeling and QoS Analysis of IEEE 802.11 Broadcast Scheme in Vehicular Ad Hoc Networks," in Proceedings of 2013 IEEE International Conference on Communications, pp. 64446448, IEEE, 2013

[2] R. Uzcategui and G. Acosta-Marum, "Wave: A tutorial," IEEE Communications Magazine, vol. 47, no. 5, pp. 126-133, 2009.

[3] H. J. Miller and S. Shaw, Geographic Information Systems for Transportation. Oxford University Press, 2001.

[4] J. Zhu and S. Roy, "MAC for Dedicated Short Range Communications in Intelligent Transport System," IEEE Communications Magazine, vol. 41, pp. 60-67, Jan. 2003

[5] "IEEE 802.11p, "Part 11: Wireless LAN Medium Access Control (MAC) and Physical Layer (PHY) Specifications Amendment 6: Wireless Access in Vehicular Environments"," July 2010.

[6] X. Ma, X. Chen, and H. H. Refai, "Performance and Reliability of DSRC Vehicular Safety Communication: A Formal Analysis," EURASIP Journal on Wireless Communications and Networking, vol. 2009, 2009.

[7] X. Ma, X. Chen, and H. H. Refai, "Unsaturated Performance of IEEE 802.11 Broadcast Service in Vehicle-to-Vehicle Networks," in Proceedings of 2007 IEEE 66th Vehicular Technology Conference, 2007. VTC-2007 Fall., pp. 1957-1961, IEEE, 2007.
[8] F. Borgonovo, A. Capone, M. Cesana, and L. Fratta, "ADHOC MAC: New MAC Architecture for Ad Hoc Networks Providing Efficient and Reliable Point-to-Point and Broadcast Services," Wireless Networks, vol. 10, no. 4, pp. 359-366, 2004.

[9] Q. Yang, J. Zheng, and L. Shen, "Modeling and Performance Analysis of Periodic Broadcast in Vehicular Ad Hoc Networks," in Proceedings of IEEE Global Telecommunications Conference (GLOBECOM 2011), (Houston, Texas), pp. 1-5, Dec. 2011.

[10] R. P. Liu, G. J. Sutton, and I. B. Collings, "A New Queueing Model for QoS Analysis of IEEE 802.11 DCF with Finite Buffer and Load," IEEE Transactions on Wireless Communications, vol. 9, pp. 2664-2675, Aug. 2010.

[11] K. Ramachandran, M. Gruteser, R. Onishi, and T. Hikita, "Experimental Analysis of Broadcast Reliability in Dense Vehicular Networks," IEEE Vehicular Technology Magazine, vol. 2, no. 2, pp. 2091-2095, 2007.

[12] Q. Xu, T. Mak, J. Ko, and R. Sengupta, "Vehicle-to-Vehicle Safety Messaging in DSRC," in Proceedings of the 1st ACM international workshop on Vehicular ad hoc networks, pp. 19-28, ACM, 2004.

[13] M. Torrent-Moreno, D. Jiang, and H. Hartenstein, "Broadcast Reception Rates and Effects of Priority Access in 802.11-Based Vehicular Ad-hoc Networks," in Proceedings of the 1st ACM international workshop on Vehicular ad hoc networks, pp. 10-18, ACM, 2004.

[14] D. Jiang, V. Taliwal, A. Meier, W. Holfelder, and R. Herrtwich, "Design of $5.9 \mathrm{GHz}$ DSRC-based Vehicular Safety Communication," IEEE Wireless Communications, vol. 13, no. 5, pp. 36-43, 2006.

[15] T. ElBatt, S. K. Goel, G. Holland, H. Krishnan, and J. Parikh, "Cooperative Collision Warning Using Dedicated Short Range Wireless Communications," in Proceedings of the 3rd international workshop on Vehicular ad hoc networks, pp. 1-9, ACM, 2006.

[16] G. Bianchi, "Performance Analysis of the IEEE 802.11 Distributed Coordination Function," IEEE Journal on Selected Areas in Communications, vol. 18 , no. 3, pp. 535-547, 2000.

[17] D. Malone, K. Duffy, and D. Leith, "Modeling the 802.11 Distributed Coordination Function in Nonsaturated Heterogeneous Conditions,' IEEE/ACM Transactions on Networking, vol. 15, no. 1, pp. 159-172, 2007.

[18] X. Ma and X. Chen, "Performance Analysis of IEEE 802.11 Broadcast Scheme in Ad Hoc Wireless LANs," IEEE Transactions on Vehicular Technology, vol. 57, no. 6, pp. 3757-3768, 2008.

[19] X. Ma and X. Chen, "Delay and Broadcast Reception Rates of Highway Safety Applications in Vehicular Ad Hoc Networks," in 2007 Mobile networking for vehicular environments, pp. 85-90, IEEE, 2007.

[20] O. Tickoo and B. Sikdar, "Queueing Analysis and Delay Mitigation in IEEE 802.11 Random Access MAC based Wireless Networks," in Proceedings of IEEE Twenty-third AnnualJoint Conference of the IEEE Computer and Communications Societies (INFOCOM'2004), (Hong Kong, China), pp. 1404-1413, Mar. 2004.

[21] M. A. Marsan, G. Taricco, and G. Tagliente, "Simulation of three MAC protocols for intelligent highway packet radio networks," Computer Communications, vol. 19, no. 12, pp. 943-953, 1996.

[22] Y.-C. Tseng, S.-Y. Ni, Y.-S. Chen, and J.-P. Sheu, "The Broadcast Storm Problem in a Mobile Ad Hoc Network," Wireless Networks, vol. 8, no. 2-3, pp. 153-167, 2002.

[23] "IEEE std 802.11-2007, "Part 11:Wireless LAN Medium Access Control (MAC) and Physical Layer (PHY) Specification",' June 2007.

[24] R. P. Liu, G. J. Sutton, and I. B. Collings, "Errata to the paper "A New Queueing Model for QoS Analysis of IEEE 802.11 DCF with Finite Buffer and Load"," IEEE Transactions on Wireless Communications, vol. 9 , pp. 1-1, Oct. 2013.

25] G. J. Sutton, R. P. Liu, and I. B. Collings, "Modelling IEEE 802.11 DCF Heterogeneous Networks with Rayleigh Fading and Capture," IEEE Transactions on Communications, vol. 61, pp. 3336-3348, Aug. 2013 


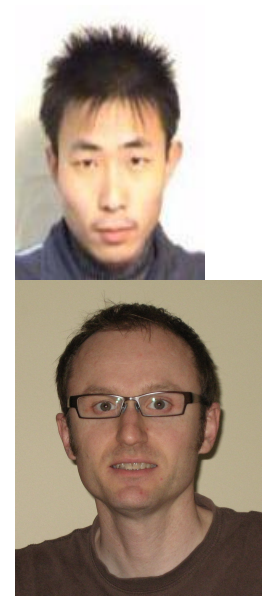

Baozhu Li received his B.E. and M.E. degrees from University of Jinan, China. He is a PhD student in Beijing University of Posts and Telecommunications, China. His research interest is vehicular ad hoc networks.

Gordon J. Sutton received a B.Sc. degree in Mathematics in 1993, B.E. degree in Systems engineering in 1995 and $\mathrm{PhD}$ in Control Theory in 1999, all from The Australian National University. He subsequently worked at ADC Australia designing optic fibre connectors and then at the Time Series Analysis Section of the Australian Bureau of Statistics. In 2006, Dr Sutton joined the Australian CSIRO as a statistician in the Quantitative Risk Management Stream of CSIRO Mathematics, Informatics and Statistics. In 2011, he joined the School of Chemistry, University of New South Wales, working in Bayesian statistics and chemometrics. Since 2015 Dr Sutton has been at the Global Big Data Technologies Centre, University of Technology Sydney, working on modelling Wi-Fi/LTE coexistence protocols. His interests include Markov processes, communications protocol modelling, process analysis and control, forecasting, signal processing, particle filters, state space modelling and Bayesian statistics.

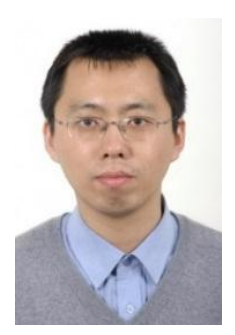

Bo $\mathbf{H u}$ is an associate professor in the State Key Laboratory of Networking and Switching Technology, Beijing University of Posts and Telecommunications (BUPT), Beijing, China. He received his PhD degree in the major of communications and information systems from BUPT in 2006. His current research interests include future wireless mobile communication systems, mobile computing and software defined networks.

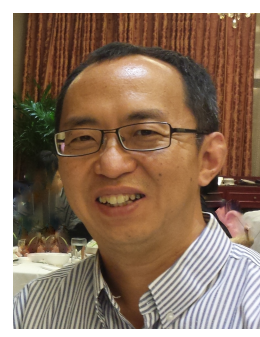

Ren Ping Liu is a Professor at the School of Computing and Communications in University of Technology Sydney, where he leads Network Security Lab. Prior to that he was a Principal Scientist at CSIRO, where he led wireless networking research activities. He specialises in protocol design and modelling, and has delivered networking solutions to a number of government agencies and industry customers. Professor Liu was the winner of Australian Engineering Innovation Award and CSIRO Chairman; ${ }^{-}$s medal. His research interests include Markov analysis and QoS scheduling in WLAN, VANET, IoT, LTE, 5G, SDN, and network security. Professor Liu has over 100 research publications, and has supervised over $30 \mathrm{PhD}$ students. Professor Liu is the founding chair of IEEE NSW VTS Chapter and a Senior Member of IEEE. He served as Technical Program Committee chair and Organising Committee chair in a number of IEEE Conferences. Ren Ping Liu received his B.E.(Hon) and M.E. degrees from Beijing University of Posts and Telecommunications, China, and the Ph.D. degree from the University of Newcastle, Australia.

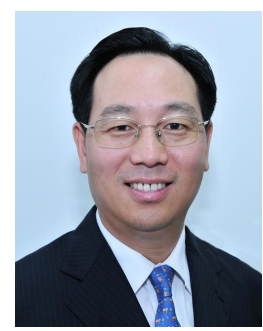

Shanzhi Chen received his Ph.D. degree from Beijing University of Posts and Telecommunications (BUPT), China, in 1997. He joined Datang Telecom Technology \& Industry Group in 1994, and has been serving as CTO since 2008. He was a member of the steering expert group on information technology of the 863 Hi-Tech Research and Development Plan of China from 1999 to 2011. He is the director of State Key Laboratory of Wireless Mobile Communications, and the board member of Semiconductor Manufacturing International Corporation (SMIC). He devoted his works to the research and development of TD-SCDMA $3 \mathrm{G}$ industrialization and
TD-LTE advanced 4G standardization. He received 2001 and 2012 National Awards for Science and Technology Progress, China, the 2015 National Award for Technological Invention, China, and the 2014 Distinguished Young Scholar Award of National Natural Science Foundation, China. His current research interests include network architecture, 5G mobile communication, and Internet of Things (IoT) 